\title{
Influence of various parameters on the a.c. diamagnetic susceptibility of Bi-based superconductors
}

\author{
M NICOLAS and J P BURGER \\ "Hydrogène \& Défauts dans les Métaux", Université Paris-Sud, Bât. 350-91405 Orsay, \\ France
}

\begin{abstract}
We present some results of a.c. susceptibility measured on pure and doped with indium and potassium $\mathrm{Bi}$-compounds. For both the $80 \mathrm{~K}$ and $110 \mathrm{~K}$ phases, we follow the $\chi^{\prime}$ and $\chi^{\prime \prime}$ variations as a function of (a) the annealing treatment, (b) the $\mathrm{Sr} / \mathrm{Ca}$ ratio and (c) the doping. Indium doping has either a positive or a negative effect on the $80 \mathrm{~K}$ material, depending on the $\mathrm{Sr} / \mathrm{Ca}$ ratio. It induces an important increase of the superconductive volume of the $110 \mathrm{~K}$ material. Effects due to potassium are opposite. The experimental results agree with the fact that $T_{c}$ goes through a maximum as the hole density increases.
\end{abstract}

Keywords. Bi superconductors; a.c. susceptibility.

\section{Introduction}

Extensive studies have been carried out on the new $\mathrm{Cu}$-based superconductor oxides due to their high transition temperature. Nevertheless these materials, in the bulk state, show behaviours which are often very different from the ideal one. Indeed they have a granular, inhomogeneous structure. Materials with not only high transition temperatures but also with narrow $\Delta T_{c}$ are difficult to obtain. However, it is now possible, to make polycrystalline compounds such as $\mathrm{Ln} \mathrm{Ba}_{2} \mathrm{Cu}_{3} \mathrm{O}_{7}[\mathrm{Ln}=$ rare earth or yttrium] by the classical solid-state reaction with relatively narrow transition. For example, one of the best compounds, $\mathrm{EuBa}_{2} \mathrm{Cu}_{3} \mathrm{O}_{7}$, has a $T_{c}=93 \mathrm{~K}$ (midpoint), $\Delta T_{c} \leqslant 2 \mathrm{~K}$ (magnetic susceptibility), $\Delta T_{\mathrm{c}} \leqslant 1 \mathrm{~K}$ (resistivity) in zero field. But; up to now, such results have not been obtained on bulk Bi-based superconductors. This family includes two groups: the first one, $\mathrm{Bi}_{2}(\mathrm{Sr}, \mathrm{Ca})_{3} \mathrm{Cu}_{2} \mathrm{O}_{x}$ with $T_{c} \simeq 70-80 \mathrm{~K}$ and the second one, $\mathrm{Bi}_{2}(\mathrm{Sr}, \mathrm{Ca})_{4} \mathrm{Cu}_{3} \mathrm{O}_{y}$ with $T_{c} \simeq 100-110 \mathrm{~K}$. The superconductive transitions are either complete but broad or narrow but partial. Indeed, as the condition of preparation of these compounds is very "pointed", they are often inhomogeneous, multi-phased, with small grains; consequently, for this latter reason, they show important effects due to Josephson coupling between the grains.

We have attempted to obtain the best Bi-samples as possible. The a.c. susceptibility measurements allowed us to distinguish between both characteristics: intragrains or intergrains effects (Nicolas and Burger 1990). Usually, the transition temperature $T_{c}$ is related to the number of holes by $\mathrm{CuO}_{2}$ plane. We report here some results where this number of holes varies with various parameters such as: oxygen content, $\mathrm{Sr} / \mathrm{Ca}$ ratio, small doping with cations of different valency.

\section{Results}

\section{$2.180 \mathrm{~K}$ compounds}

(i) Undoped samples: Two compounds have been studied with a different $\mathrm{Sr} / \mathrm{Ca}$ ratio: the [4334]-phase with $\mathrm{Sr} / \mathrm{Ca}=1$ and the [2212]-one with $\mathrm{Sr} / \mathrm{Ca}=2$. We have been 
able to obtain relatively good samples with a narrow transition compared to the yet known results. For both samples, $T_{c}$ goes through a maximum as the annealing temperatures increases, but their behaviour is opposite as regards oxygen: for $\mathrm{Sr} / \mathrm{Ca}=1, T_{\mathrm{c}}$ is maximum with an additional oxygen whereas for $\mathrm{Sr} / \mathrm{Ca}=2$, a release of oxygen is necessary to reach a $T_{c}$ maximum. The variations of $\chi^{\prime}$ and $\chi^{\prime \prime}$ with the a.c. field show that the grains remain small compared to the near $T_{c}$ penetration depth.

(ii) Doped samples: The trivalent In and the monovalent $\mathrm{K}^{+}$have been checked. In the case of $\mathrm{In}^{+++}$-doping, $T_{c}$ decreases a little and the superconductive volume diminishes quickly when $\mathrm{Sr} / \mathrm{Ca}=1$ whereas the effect is opposite with respect to $T_{c}$ in the case of $\mathrm{Sr} / \mathrm{Ca}=2$. Indeed $T_{c}$ increases, goes through a maximum and decreases with In-concentration. The superconductive volume does not vary and remains maximum for the studied concentrations $(0 \leqslant x \leqslant 0 \cdot 3)$ (Nicolas et al 1990). Doping with potassium induces opposite effects on the [2212] phase.

All these results can be well understood if one considers that, as shown previously for $\mathrm{La}_{2-x} \mathrm{Sr}_{x} \mathrm{CuO}_{4}$ (Torrance et al 1988) and for $\mathrm{Bi}_{2}(\mathrm{Sr}, \mathrm{Ca})_{3} \mathrm{Cu}_{2} \mathrm{O}_{8 \pm \delta}$ (Buckley et al 1988; Koike et al 1989), the variations of $T_{c}$ as a function of $p$, the hole density, presents a maximum. In the case of the $\mathrm{Bi}$-compound, the density of holes would be governed by the $\mathrm{Sr} / \mathrm{Ca}$ ratio. When $\mathrm{Sr} / \mathrm{Ca}=1, p$ is such that $T_{c}$ is not at the maximum value (before the maximum) and it is necessary to oxidize a little to reach $T_{c}$ maximum whereas for $\mathrm{Sr} / \mathrm{Ca}=2, p$ is such that $T_{c}$ is situated after the maximum and it is necessary to deoxidize in order to reach $T_{\max }$ As $\mathrm{In}^{+++}$decreases the density of holes if substituted the $\mathrm{Ca}^{++}$and $\mathrm{K}^{+}$increases it if substituted to $\mathrm{Bi}^{++}$, the above mentioned opposite behaviours can be well explained.

\section{$2.2110 \mathrm{~K}$ compound}

It corresponds to an additional $\mathrm{CaCuO}_{2}$ plane in the structure of $\mathrm{Bi}_{2} \mathrm{Sr}_{2} \mathrm{CaCu}_{2} \mathrm{O}_{8}$. Its formula would be $\mathrm{Bi}_{2}(\mathrm{SrCu})_{4} \mathrm{Cu}_{3} \mathrm{O}_{10}$. The monophased system is very difficult to obtain. In-doping decreases $T_{c}$ slightly $(110 \mathrm{~K}-106 \mathrm{~K})$ but increases strongly the superconductive volume. K-doping provokes an opposite behaviour.

\section{Conclusion}

We have been able to prepare nearly-pure $\mathrm{Bi}$-phases. The $\chi^{\prime}$ and $\chi^{\prime \prime}$ measurements allow us to determine various factors such as homogeneity, grain size, coupling between grains etc. Our most important result is the significant increase of the $110 \mathrm{~K}$-phase volume by In-doping.

\section{References}

Buckley R G 1988 Physica C156 629

Koike Y et al 1989 Physica C159 105

Nicolas M and Burger J P 1990 (to be published)

Nicolas M, Burger J P and Negri J 1990 (to be published)

Torrance J B et al 1988 Phys. Rev. Lett. 611127 